\title{
The HIT-SCIR System for End-to-End Parsing of Universal Dependencies
}

\author{
Wanxiang Che, Jiang Guo, Yuxuan Wang, Bo Zheng \\ Huaipeng Zhao, Yang Liu, Dechuan Teng and Ting Liu \\ Center for Social Computing and Information Retrieval \\ Harbin Institute of Technology, Harbin, China 150001 \\ \{wxche, jguo, yxwang, bzheng, hpzhao,yliu, dcteng, tliu\}@ir.hit.edu.cn
}

\begin{abstract}
This paper describes our system (HITSCIR) for the CoNLL 2017 shared task: Multilingual Parsing from Raw Text to Universal Dependencies. Our system includes three pipelined components: tokenization, Part-of-Speech (POS) tagging and dependency parsing. We use character-based bidirectional long shortterm memory (LSTM) networks for both tokenization and POS tagging. Afterwards, we employ a list-based transitionbased algorithm for general non-projective parsing and present an improved StackLSTM-based architecture for representing each transition state and making predictions.
\end{abstract}

Furthermore, to parse low/zero-resource languages and cross-domain data, we use a model transfer approach to make effective use of existing resources. We demonstrate substantial gains against the UDPipe baseline, with an average improvement of $3.76 \%$ in LAS of all languages. And finally, we rank the 4th place on the official test sets.

\section{Introduction}

Our system for the CoNLL 2017 shared task (Zeman et al., 2017) is a pipeline which includes three cascaded modules, tokenization, Part-of-Speech (POS) tagging and dependency parsing.

- Tokenization. This module includes two components, the sentence segmenter and the word segmenter which recognize the sentence and word boundaries respectively (Section 2.1).
- POS tagging. We focus mainly on universal POS tags, and don't use language-specific POS as well as other morphological features (Section 2.2).

- Dependency parsing. To handle the nonprojective dependencies in most of the languages (or treebanks) provided in the task, we employ the list-based transition parsing algorithm (Choi and McCallum, 2013), equipped with an improved Stack-LSTMbased model for representing the transition states, i.e., configurations (Section 2.3).

We mainly concentrate on parsing in this task, and make use of UDPipe (v1.1) (Straka et al., 2016a) for most of the pre-processing steps. However, our preliminary experiments showed that the UDPipe tokenizer and POS tagger perform rather poorly in some languages and specific domains. Therefore, we develop our own tokenizer and POS tagger for a subset of languages.

To deal with the parallel test sets (crossdomain) and low/zero-resource languages, we adopt the neural transfer approaches proposed in our previous studies (Guo et al., 2015, 2016) to encourage knowledge transfer across different but related languages or treebanks.

Experiments on 81 test sets demonstrate that our system (HIT-SCIR: software4) obtains an average improvement of $3.76 \%$ in LAS as compared with the UDPipe baseline, and ranks the 4th place in this task.

\section{System Architecture}

\subsection{Tokenization}

\subsubsection{Sentence Segmentation}

We develop our own sentence segmentation models for the languages which have white spaces as token separators and on which UDPipe doesn't 


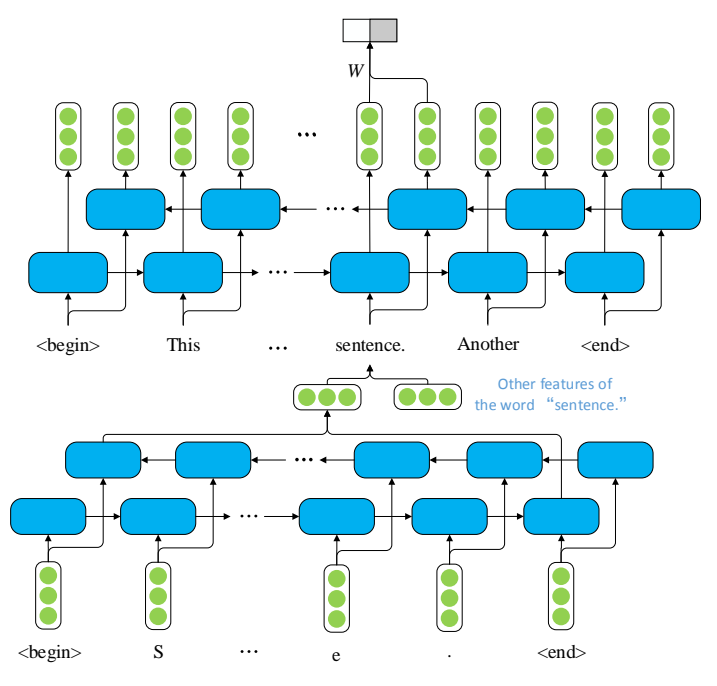

Figure 1: The hierarchical Bi-LSTM model for sentence segmentation.

perform well. We formalize the sentence segmentation process as a binary classification problem, that is to classify each token as either the end of a sentence or not. We notice that characterlevel information is critical for sentence segmentation, since texts are not tokenized yet in the current phase. Therefore, we develop a hierarchical LSTM-based model, as illustrated in Figure 1, in which characters in each token are composed using a character-based bidirectional LSTM (BiLSTM) network and then concatenated with additional token-level features (e.g., token embedding, the first character of this token, etc.) and passed through a token-level Bi-LSTM. The hidden states of the token-level Bi-LSTM are finally used for classification through a softmax layer.

We follow the strategy of the UDPipe tokenizer (Straka et al., 2016a) and employ a sliding window to incrementally segment a document into sentences.

In addition, we notice that for certain treebanks (e.g., la_ittb and cs_cltt), some punctuation-related rules derived from the training data can be highly effective. To be more specific, some punctuations that appear as the end of a sentence with high probability will be used directly for determining sentence boundaries. Therefore, we develop additional rule-based systems for these data instead of using the neural models as describe above.

\subsubsection{Word Segmentation}

We develop our own word segmentation models particularly for languages which do not have ex-

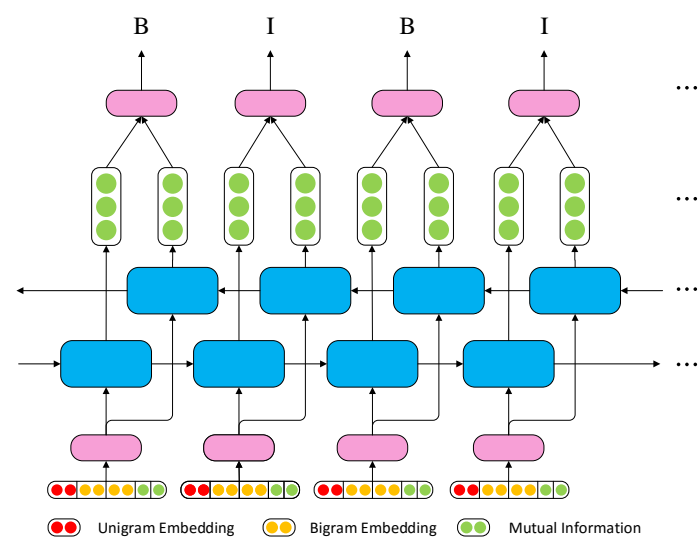

Figure 2: The word segmentation model. 'B' denotes the beginning position and ' $\mathrm{I}$ ' denotes the middle or ending positions of a word.

plicit word boundary markers, i.e., white spaces, including Chinese, Japanese and Vietnamese. ${ }^{1}$

Our word segmentation model is also built on Bi-LSTM networks, and incorporates rich statistics-based features gathered from large-scale unlabeled data. Specifically, we utilize features like character-unigram embeddings, characterbigram embeddings and the pointwise mutual information (Liang, 2005) (PMI) of adjacent characters. Formally, the input of our model at each time step $t$ can be computed as:

$$
z_{t}=\left[U_{t} ; B_{t-1} ; B_{t} ; \operatorname{PMI}\left(c_{t-1}, c_{t}\right) ; \operatorname{PMI}\left(c_{t}, c_{t+1}\right)\right]
$$

$$
x_{t}=\max \left\{0, W z_{t}+b\right\}
$$

where $U_{t}$ and $B_{t}$ denote the unigram embedding and bigram embedding respectively at position $t$ and PMI denotes the pointwise mutual information between two characters.

The PMI values are computed through:

$$
\operatorname{PMI}\left(c_{1}, c_{2}\right)=\log \frac{p\left(c_{1} c_{2}\right)}{p\left(c_{1}\right) p\left(c_{2}\right)}
$$

where $c_{1}$ and $c_{2}$ are two characters, $p\left(c_{1}\right), p\left(c_{2}\right)$ and $p\left(c_{1} c_{2}\right)$ are counted on the raw data provided by the shared task. $p(s)$ denotes the probability string $s$ appears in the raw data. We scale PMI

\footnotetext{
${ }^{1}$ Vietnamese requires word segmentation because white spaces occur both inter- and intra-words. When segmenting Vietnamese, white space-separated tokens are used as inputs, rather than characters as in Chinese and Japanese. In addition, we don't consider Korean here since the Korean input texts have already been segmented in the corpus provided by the task.
} 


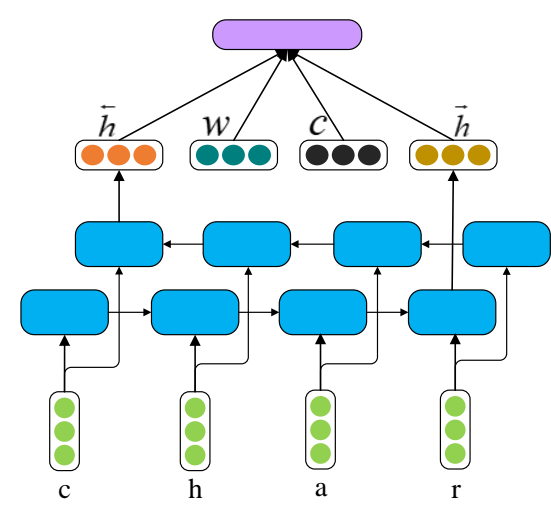

Figure 3: Structure of the character-based composition model for learning word representations. ' $w$ ' denotes the pre-trained word embedding, ' $c$ ' denotes the Brown cluster embedding.

with their Z-scores, the Z-score of a PMI value $x$ is $\frac{x-\mu}{\sigma}$, where $\mu$ and $\sigma$ are the mean and standard deviation of the PMI distribution, respectively.

Figure 2 shows the architecture of our word segmentation model.

The character-unigram embeddings and character-bigram embeddings are obtained using word2vec (Mikolov et al., 2013) on the raw data.

\subsection{Part-of-Speech Tagging}

The UDPipe POS tagger is trained using averaged perceptron with feature engineering. In our system, we use a model similar to the one for sentence segmentation (Section 2.1.1), i.e., a hierarchical Bi-LSTM model which outperforms UDPipe on most of datasets with much fewer features. Concretely, each word is modeled using a characterbased Bi-LSTM, so that word prefix and suffix features can be effectively incorporated, which is particularly important for morphologically rich languages. In addition, modeling from characters alleviates the problem of Out-of-Vocabulary (OOV) words.

The character-based compositional embedding of each word is then concatenated with a pretrained word embedding and a Brown cluster embedding, resulting in the final word representation which is fed as input of a word-level Bi-LSTM for POS tagging. Formally,

$$
x=\max \{0, W[\vec{h} ; \overleftarrow{h} ; w ; c]+q\}
$$

Figure 3 illustrates the structure of the character-based composition model.

\subsection{Dependency Parsing}

The transition-based dependency parsing algorithm with a list-based arc-eager transition system proposed by Choi and McCallum (2013) is used in our parser. We base our parser mainly on the Stack-LSTM model proposed by Dyer et al. (2015), where three Stack-LSTMs are utilized to incrementally obtain the representations of the buffer $\beta$, the stack $\sigma$ and the transition action sequence $A$. In addition, a dependency-based Recursive Neural Network (RecNN) is used to compute the partially constructed tree representation. However, compared with the arc-standard algorithm (Nivre, 2004) used by Dyer et al. (2015), the list-based arc-eager transition system has an extra component in each configuration, i.e., the deque $\delta$. So we use an additional Stack-LSTM to learn the representation of $\delta$. More importantly, we introduce two LSTM-based techniques, namely Bi-LSTM Subtraction and Incremental Tree-LSTM (explained below) for modeling the buffer and sub-tree representations in our model.

The pre-trained word embedding (100dimensional), Brown cluster embedding (100dimensional), along with a 100-dimensional randomly initialized word embedding updated while training, ${ }^{2}$ and a 50-dimensional embedding for UPOS are concatenated and passed through a non-linear layer to obtain the representation of each word.

Representations of the four components in our transition system are concatenated and passed through a hidden layer to obtain the representation of the parsing state at time $t$ :

$$
e_{t}=\max \left\{0, W\left[s_{t} ; b_{t} ; p_{t} ; a_{t}\right]+d\right\}
$$

where $s_{t}, b_{t}, p_{t}$ and $a_{t}$ are the representation of $\sigma, \beta, \delta$ and $A$ respectively. $d$ is the bias. $e_{t}$ is finally used to compute the probability distribution of possible transition actions at time $t$ through a softmax layer. Figure 4 shows the architecture.

\subsubsection{Bi-LSTM Subtraction}

We regard the buffer as a segment and use the subtraction between LSTM hidden vectors of the segment head and tail as its representation. To include the information of words out of the buffer, we apply subtraction on bidirectional LSTM representations over the whole sentence (Wang et al.,

\footnotetext{
${ }^{2}$ Unfortunately we did not have access to enough raw text of Gothic, thus no pre-trained word embedding nor Brown cluster is utilized for it
} 


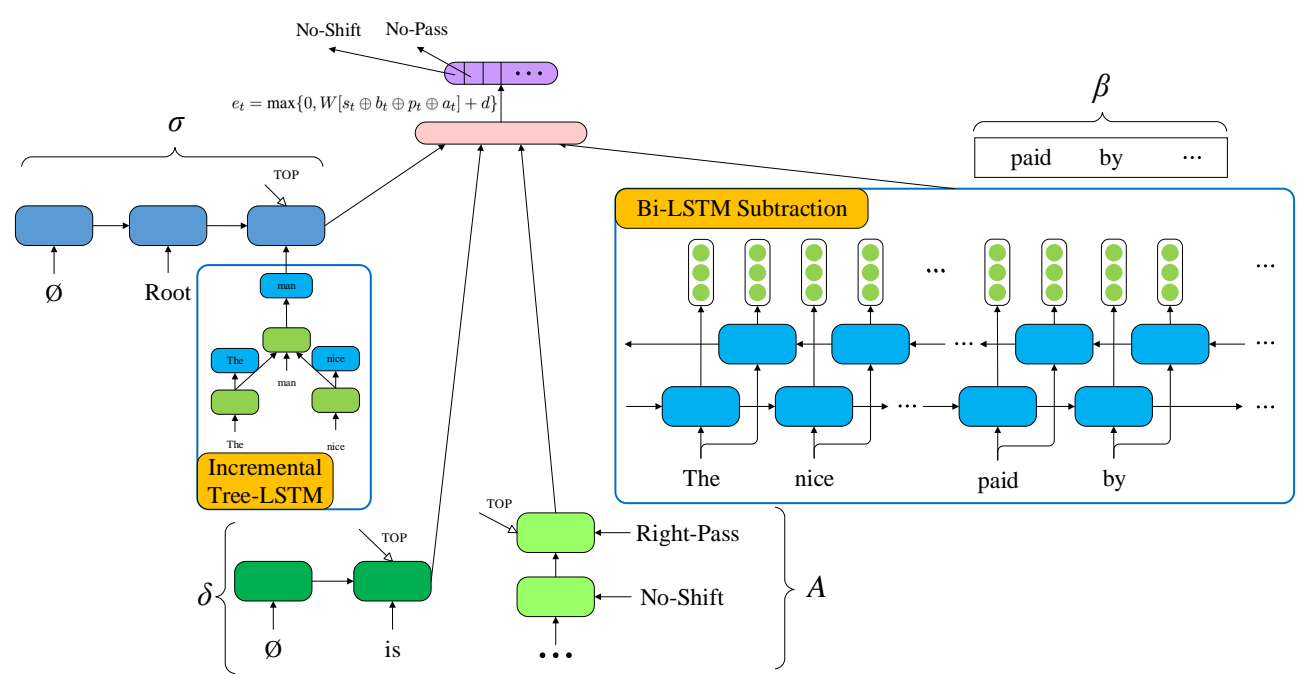

Figure 4: Example transition state representation based on LSTMs. The buffer $\beta$ is represented by Bi-LSTM Subtraction, the sub-trees are computed by Incremental Tree-LSTM.

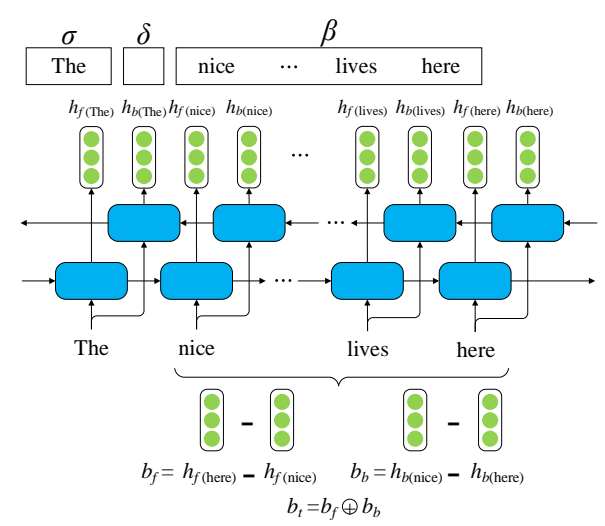

Figure 5: Illustration of Bi-LSTM Subtraction for buffer representation learning. $h_{f}(*)$ and $h_{b}(*)$ indicate the hidden vectors of forward and backward LSTM respectively. $b_{t}$ is the resulting buffer representation.

2016; Kiperwasser and Goldberg, 2016; Cross and Huang, 2016), thus called Bi-LSTM Subtraction.

The forward and backward subtractions are calculated independently, i.e., $b_{f}=h_{f}(l)-h_{f}(f)$ and $b_{b}=b_{b}(f)-b_{b}(l)$, where $h_{f}(f)$ and $h_{f}(l)$ are the hidden vectors of the first and the last words in the forward LSTM, $h_{b}(f)$ and $h_{b}(l)$ are the hidden vectors of the first and the last words in the backward LSTM. Then $b_{f}$ and $b_{b}$ are concatenated as the buffer representation. As illustrated in Figure 5, the forward and backward subtractions for the buffer are $b_{f}=h_{f}($ here $)-h_{f}($ nice $)$ and $b_{b}=h_{b}($ nice $)-h_{b}($ here $)$ respectively.

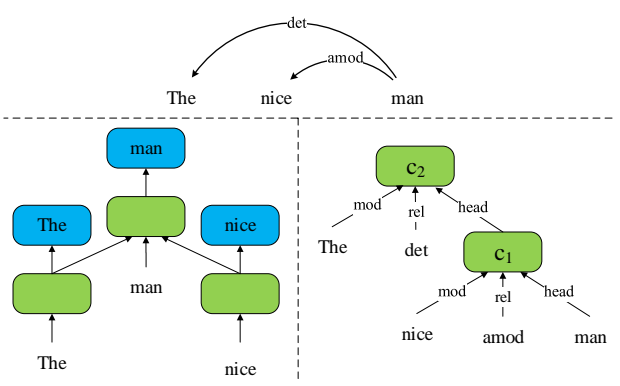

Figure 6: Representations of a dependency subtree (above) computed by Tree-LSTM (left) and dependency-based RecNN (right).

\subsubsection{Incremental Tree-LSTM}

We use a Tree-LSTM (Tai et al., 2015; Zhu et al., 2015) in our parser to model the sub-trees during parsing. The example in Figure 6 shows the differences between RecNN (Dyer et al., 2015) and Tree-LSTM. In RecNN, the representation of a sub-tree is computed by recursively combining head-modifier pairs. Whereas in Tree-LSTM, a head is combined with all of its modifiers simultaneously in each LSTM unit.

However, our implementation of Tree-LSTM is different from the conventional one. Unlike traditional bottom-up Tree-LSTMs in which each head and all of its modifiers are combined simultaneously, the modifiers are found incrementally during our parsing procedure. Therefore, we propose Incremental Tree-LSTM, which obtains sub-tree representations incrementally. To be more specific, each time a dependency arc is generated, 
we collect representations of all the found modifiers of the head and combine them along with the embedding of the head as the representation of the sub-tree. The original embedding rather than the current representation of the head is utilized to avoid the reuse of modifier information, since the current representation of the head contains information of its modifiers found previously.

\subsubsection{Parser Ensembling}

For a majority of languages, we found that the parsing performance can be improved by simply integrating two separately trained models. More specifically, for each language two models with different random seeds are trained separately. While predicting, in each state, both models are used to calculate the scores for valid transitions under this configuration as described above. Then the score distributions computed by two models are summed to get the final scores for the valid transitions, among which the one with the highest score will be taken as the next transition.

\section{Transfer Parsing across Domains and Languages}

\subsection{Cross-Domain Transfer}

For 15 out of 45 languages presented in the task, multiple treebanks from different domains are provided. To exploit the benefits from these crossdomain data, we use a simple inductive transfer approach which has two stages:

1. Multiple treebanks of each language are combined to train an unified parser.

2. The unified parser is then fine-tuned on the training treebank of each domain, to obtain target domain-specific parsers.

In practice, for each language considered here, we treat the largest treebank as our source-domain data, and the rest as target-domain data. Only target-domain models are fine-tuned from the unified parser, while the source-domain parser is trained separately using the source treebank alone.

For the new parallel test sets in test phase, we simply use the model trained on source-domain data, without any assumption on the target domain.

\subsection{Cross-Lingual Transfer}

We consider the languages which have less than 900 sentences in the training treebank as low-

\begin{tabular}{c|ccccc}
\hline Target & hu & uk & qa & ug & kk \\
Source & fi_ftb & ru_syntagrus & en & tr & tr \\
\hline
\end{tabular}

Table 1: Cross-lingual transfer settings for lowresource target languages.

resource, and employ the cross-lingual model transfer approach described in Guo et al. (2015, 2016) to benefit from existing resource-rich languages.

The low-resource languages here include Ukrainian (uk), Irish (ga), Uyghur (ug) and Kazakh (kk). We determine their source language (treebank) according to the language families they belong to and their linguistic typological similarity. Specifically, the transfer setting is shown in Table 1.

The transfer approach is similar to crossdomain transfer as described above, with one important difference. Here, we use cross-lingual word embeddings and Brown clusters derived by the robust projection approach (Guo et al., 2015) when training the unified parser, to encourage knowledge transfer across languages at lexical level. Specifically, for each source and target language pair $\langle\mathrm{src}$, tgt $\rangle$, we derive an alignment matrix $A_{t g t \mid s r c}$ from a collected bilingual parallel corpus, where each element $A_{t g t \mid s r c}(i, j)$ is the normalized count of alignments between corresponding words in their vocabularies:

$$
A_{t g t \mid s r c}(i, j)=\frac{\#\left(V_{t g t}^{(i)} \leftrightarrow V_{s r c}^{(j)}\right)}{\sum_{k} \#\left(V_{t g t}^{(i)} \leftrightarrow V_{s r c}^{(k)}\right)}
$$

Given a pre-trained source language word embedding matrix $E_{s r c}$, the resulting word embedding matrix for the target language can be simply computed as:

$$
E_{t g t}=A_{t g t \mid s r c} \cdot E_{s r c}
$$

Therefore, the embedding of each word in the target language is the weighted average of the embeddings of its translation words in our bilingual parallel corpus.

The cross-lingual Brown clusters are obtained using the PROJECTED clustering approach described in (Täckström et al., 2012), which assigns a target word to the cluster with which it is most often aligned:

$$
c\left(w_{i}^{t g t}\right)=\underset{k}{\arg \max } \sum_{j} A_{t g t \mid s r c}(i, j) \cdot \mathbb{1}\left[c\left(w_{j}^{s r c}\right)=k\right]
$$




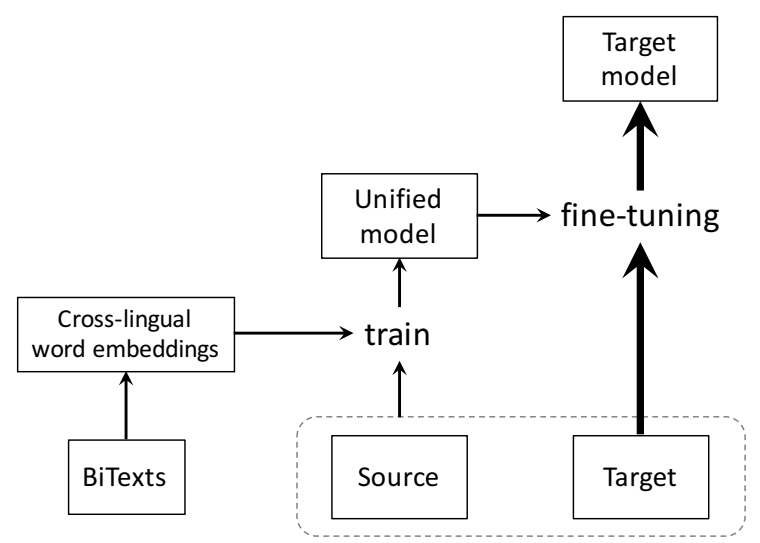

Figure 7: The cross-lingual transfer approach.

\begin{tabular}{c|cccc}
\hline Target & bxr & kmr & sme & hsb \\
Source & tr \& ug \& kk & fa & fi_ftb \& fi & cs \\
\hline
\end{tabular}

Table 2: Cross-lingual delexicalized transfer settings for surprise languages.

After that, target language-specific parsers are obtained through fine-tuning on their own treebanks. Figure 7 illustrates the flow of our transfer approach.

For the surprise languages in the final test phase, we use the transfer settings in Table 2. We use multi-source delexicalized transfer for surprise language parsing, considering that bilingual parallel data which is required for obtaining crosslingual word embeddings is not available for these languages.

\section{Experiments}

We first describe our experiment setups and strategies for processing different languages (treebanks) in each module. Then we present the results and analysis.

\subsection{Experimental Settings}

\subsubsection{Model Selection Strategies}

For sentence segmentation, we apply our own models for a subset of languages on which UDPipe yields poor performance, and use UDPipe for the rest languages. ${ }^{3}$ Specifically, we use the rulebased model for la_ittb and cs_cltt, ${ }^{4}$ and use the BiLSTM-based model (Figure 1) for sk, en, en_lines, fi_ftb, got, nl_lassysmall, grc_proiel, la_ittb, cu,

\footnotetext{
${ }^{3}$ We use the same hyper-parameter settings as provided by the organizers to train the UDPipe models.

${ }^{4}$ However, the rule-based model does not yield good performance on the two test sets. We suggest that the rules we use are overfitting the development sets to some degree.
}

la_proiel, da and sl_sst. For word segmentation, we use our Bi-LSTM-based model for zh, ja, ja_pud and vi, which don't have explicit word boundary markers, i.e., white spaces.

We use our own POS taggers for all of the languages, except for the surprise languages, which we rely on UDPipe for all pre-processing steps.

Our strategies for parsing are shown in Table 3. We determine the optimal parser (single, ensemble or transfer) for each treebank according to the performance on the development data.

\subsubsection{Data and Tools}

We use the provided 100-dimensional multilingual word embeddings ${ }^{5}$ in our tokenization, POS tagging and parsing models, and use the Wikipedia and CommonCrawl data for training Brown clusters. The number of clusters is set to 256 .

For cross-lingual transfer parsing of lowresource languages, we use parallel data from OPUS to derive cross-lingual word embeddings. ${ }^{6}$ The fast_align toolkit (Dyer et al., 2013) is used for word alignment. ${ }^{7}$

We use the Dynet toolkit for the implementation of all our neural models. ${ }^{8}$

\subsection{Effects of Different Parts in Dependency Parsing}

We conduct experiments on the development sets of 4 treebanks to investigate the contributions of the two architectures we proposed (i.e., the Incremental Tree-LSTM and the Bi-LSTM Subtraction) and the Brown cluster. The LAS of different experiment settings are presented in Table 4. Results show that Brown clusters and both architectures help to improve the parsing performance in most situations. And the ensemble method we eventually choosed which incorporated the two architectures as well as Brown clusters and utilized two models for predicting yield the best performance.

\subsection{Effect of Transfer Parsing}

To investigate the effect of transfer parsing on cross-domain and cross-lingual data, we compare our transferred system with the supervised system on a subset of treebanks. Evaluation is conducted

\footnotetext{
${ }^{5}$ lindat.mff.cuni.cz/repository/xmlui/ handle/11234/1-1989

${ }^{6}$ opus.lingfil.uu.se

${ }^{7}$ https://github.com/clab/fast_align

${ }^{8}$ github.com/clab/dynet
} 


\begin{tabular}{l|l}
\hline Strategy & ltcode \\
\hline Single & cs_cac, bg, ja, he \\
\hline Ensemble (2) & $\begin{array}{l}\text { cs, ru_syntagrus, la_ittb, fi_ftb, grc_proiel, es_ancora, es, de, hi, ca, } \\
\text { en, fi, sk, ro, hr, pl, ar, eu, fa, id, ko, da, sv, cu, ur, zh, tr, got, sv_lines, } \\
\text { lv, gl, et, el, vi, hu }\end{array}$ \\
\hline Cross-domain Transfer & $\begin{array}{l}\text { no_bokmaal, no_nynorsk, la, la_proiel, grc, pt,pt_br, sl, sl_sst, nl, } \\
\text { nl_lassysmall, en_lines, en_partut, fr, fr_sequoia, fr_partut, it, it_partut } \\
\text { gl_treegal, cs_cltt, ru }\end{array}$ \\
\hline Cross-lingual Transfer & uk, ga, ug, kk \\
\hline Delexicalized Transfer & bxr, kmr, sme, hsb \\
\hline
\end{tabular}

Table 3: Model selection strategies for all treebanks. ${ }^{\dagger}$ it_partut is excluded from the final test sets. But it's used in our transfer parsing as a source treebank.

\begin{tabular}{l|cccc}
\hline Settings & cs & de & en & ko \\
\hline Baseline & 86.79 & 80.08 & 81.87 & 67.21 \\
B & 87.51 & 80.01 & 82.48 & 68.19 \\
T & 87.43 & 80.01 & 82.24 & 68.29 \\
B + T & 87.67 & 80.24 & 82.73 & 68.34 \\
B + T + C & 87.62 & 81.45 & 83.37 & 70.24 \\
\hline Ensemble (2) & $\mathbf{8 8 . 5 2}$ & $\mathbf{8 1 . 9 2}$ & $\mathbf{8 3 . 9 9}$ & $\mathbf{7 1 . 3 8}$ \\
\hline
\end{tabular}

Table 4: Experiment results (LAS) on development sets with different settings. B: BiLSTM Subtraction, T: Incremental Tree-LSTM, C: Brown cluster. Ensemble is produced with models we eventually submitted.

on the development data or through 5-fold crossvalidation when development data is not available. Results are shown in Table 5 and 6 respectively. We can see that both cross-domain and cross-lingual transfer parsing improve over the supervised systems significantly.

\subsection{Results}

The overall results of our end-to-end universal parsing system on 81 test treebanks are shown in Table 7, with comparison to the UDPipe baseline models. We obtain substantial gains over UDPipe on 76 out of 81 treebanks, with $3.76 \%$ improvements in average LAS. It spent about 9 hours to evaluate all of 81 test sets end-to-end and needed up to 4GB memory on the TIRA virtual machine.

\subsection{Post-Evaluation}

We realized a small problem in our implementation of the word segmentation models after official evaluation. After revision, we re-evaluated our models on the four test treebanks: zh, vi, ja

\begin{tabular}{l|ll|ll}
\hline \multirow{2}{*}{ Itcode } & \multicolumn{2}{|c}{ Supervised } & \multicolumn{2}{c}{ Transfer } \\
& UAS & LAS & UAS & LAS \\
\hline cs_cltt & 81.23 & 77.80 & 85.38 & 83.08 \\
en_lines & 82.30 & 78.44 & 83.65 & 79.75 \\
en_partut & 82.66 & 78.72 & 85.97 & 82.08 \\
fr_sequoia & 88.68 & 86.67 & 89.46 & 87.79 \\
la_proiel & 78.77 & 73.18 & 79.67 & 74.46 \\
no_bokmaal & 89.76 & 87.50 & 90.49 & 88.37 \\
no_nynorsk & 88.39 & 85.84 & 89.41 & 87.08 \\
nl_lassysmall & 86.11 & 82.65 & 87.39 & 84.02 \\
pt_br & 91.59 & 89.65 & 91.93 & 90.16 \\
\hline Average & 85.50 & 82.27 & $\mathbf{8 7 . 0 4}$ & $\mathbf{8 4 . 0 9}$ \\
\hline
\end{tabular}

Table 5: Effects of cross-domain transfer parsing on a subset of development sets.

and ja_pud. The post-evaluation results are shown in Table 8. On zh, vi and ja_pud, we outperform the rank-1 system significantly. We can see that the performance of word segmentation is crucial for the pipeline system.

\section{Conclusion and Future Work}

Our CoNLL-2017 system on end-to-end universal parsing includes three cascaded modules, tokenization, POS tagging and dependency parsing. We develop effective neural models for each task, with particular utilization of bidirectional LSTM networks. Furthermore, we use transfer parsing approaches for cross-domain and crosslingual adaption, that can effectively exploit resources from multiple treebanks. We obtain significant improvements against the UDPipe baseline systems on most of the test sets, and obtain the 4th place in the final evaluation. 


\begin{tabular}{l|cc|cc}
\hline \multirow{2}{*}{ Itcode } & \multicolumn{2}{|c}{ Supervised } & \multicolumn{2}{c}{ Transfer } \\
& UAS & LAS & UAS & LAS \\
\hline uk & 78.75 & 72.47 & 86.27 & 80.92 \\
ga & 76.66 & 67.08 & 80.83 & 73.44 \\
ug & 58.53 & 38.32 & 67.19 & 52.23 \\
\hline Average & 71.31 & 59.29 & $\mathbf{7 8 . 1 0}$ & $\mathbf{6 8 . 8 6}$ \\
\hline
\end{tabular}

Table 6: Effects of cross-lingual transfer parsing on ug uk and ga. 5-fold cross-validation is used for evaluation.

\section{Credits}

There are a few references we would like to give proper credit, especially to data providers: the core Universal Dependencies paper from LREC 2016 (Nivre et al., 2016), the UD version 2.0 datasets (Nivre et al., 2017b,a), the baseline UDPipe models (Straka et al., 2016b), the baseline SyntaxNet models (Weiss et al., 2015) and the evaluation platform TIRA (Potthast et al., 2014).

\section{Acknowledgments}

This work was supported by the National Key Basic Research Program of China via grant 2014CB340503 and the National Natural Science Foundation of China (NSFC) via grant 61300113 and 61632011.

\section{References}

Jinho D. Choi and Andrew McCallum. 2013. Transition-based dependency parsing with selectional branching. In Proc. of ACL. pages 10521062.

James Cross and Liang Huang. 2016. Incremental parsing with minimal features using bi-directional 1stm. In Proc. of ACL. pages 32-37.

Chris Dyer, Miguel Ballesteros, Wang Ling, Austin Matthews, and Noah A. Smith. 2015. Transitionbased dependency parsing with stack long shortterm memory. In Proc. of ACL and IJCNLP. pages 334-343.

Chris Dyer, Victor Chahuneau, and Noah A. Smith. 2013. A simple, fast, and effective reparameterization of ibm model 2. In NAACL. Association for Computational Linguistics, Atlanta, Georgia, pages 644-648.

Jiang Guo, Wanxiang Che, David Yarowsky, Haifeng Wang, and Ting Liu. 2015. Cross-lingual dependency parsing based on distributed representations. In Proceedings of the 53rd Annual Meeting of the Association for Computational Linguistics and the 7th International Joint Conference on Natural Language Processing (Volume 1: Long Papers). pages 1234-1244.

Jiang Guo, Wanxiang Che, David Yarowsky, Haifeng Wang, and Ting Liu. 2016. A representation learning framework for multi-source transfer parsing. In $A A A I$. pages 2734-2740.

Eliyahu Kiperwasser and Yoav Goldberg. 2016. Simple and accurate dependency parsing using bidirectional lstm feature representations. TACL 4:313327.

Percy Liang. 2005. Semi-supervised learning for natural language. Master thesis, Massachusetts Institute of Technology.

Tomas Mikolov, Kai Chen, Greg Corrado, and Jeffrey Dean. 2013. Efficient estimation of word representations in vector space. International Conference on Learning Representations (ICLR) Workshop .

Joakim Nivre. 2004. Incrementality in deterministic dependency parsing. In Proc. of the Workshop on Incremental Parsing: Bringing Engineering and Cognition Together. pages 50-57.

Joakim Nivre, Željko Agić, Lars Ahrenberg, Lene Antonsen, Maria Jesus Aranzabe, Masayuki Asahara, Luma Ateyah, Mohammed Attia, Aitziber Atutxa, Elena Badmaeva, Miguel Ballesteros, Esha Banerjee, Sebastian Bank, John Bauer, Kepa Bengoetxea, Riyaz Ahmad Bhat, Eckhard Bick, Cristina Bosco, Gosse Bouma, Sam Bowman, Aljoscha Burchardt, Marie Candito, Gauthier Caron, Gülşen Cebirolu Eryiit, Giuseppe G. A. Celano, Savas Cetin, Fabricio Chalub, Jinho Choi, Yongseok Cho, Silvie Cinková, Çar Çöltekin, Miriam Connor, Marie-Catherine de Marneffe, Valeria de Paiva, Arantza Diaz de Ilarraza, Kaja Dobrovoljc, Timothy Dozat, Kira Droganova, Marhaba Eli, Ali Elkahky, Tomaž Erjavec, Richárd Farkas, Hector Fernandez Alcalde, Jennifer Foster, Cláudia Freitas, Katarína Gajdošová, Daniel Galbraith, Marcos Garcia, Filip Ginter, Iakes Goenaga, Koldo Gojenola, Memduh Gökrmak, Yoav Goldberg, Xavier Gómez Guinovart, Berta Gonzáles Saavedra, Matias Grioni, Normunds Grūzítis, Bruno Guillaume, Nizar Habash, Jan Hajič, Jan Hajič jr., Linh Hà M, Kim Harris, Dag Haug, Barbora Hladká, Jaroslava Hlaváčová, Petter Hohle, Radu Ion, Elena Irimia, Anders Johannsen, Fredrik Jørgensen, Hüner Kaşkara, Hiroshi Kanayama, Jenna Kanerva, Tolga Kayadelen, Václava Kettnerová, Jesse Kirchner, Natalia Kotsyba, Simon Krek, Sookyoung Kwak, Veronika Laippala, Lorenzo Lambertino, Tatiana Lando, Phng Lê Hng, Alessandro Lenci, Saran Lertpradit, Herman Leung, Cheuk Ying Li, Josie Li, Nikola Ljubešić, Olga Loginova, Olga Lyashevskaya, Teresa Lynn, Vivien Macketanz, Aibek Makazhanov, Michael Mandl, Christopher Manning, Ruli Manurung, Cătălina Mărănduc, David Mareček, Katrin Marheinecke, Héctor Martínez Alonso, André Martins, 


\begin{tabular}{|c|c|c|c|c|c|c|c|c|c|}
\hline \multirow{2}{*}{ Itcode } & \multicolumn{2}{|c|}{ UDPipe 1.1} & \multicolumn{2}{|c|}{ Ours } & \multirow{2}{*}{ Itcode } & \multicolumn{2}{|c|}{ UDPipe 1.1} & \multicolumn{2}{|c|}{ Ours } \\
\hline & UAS & LAS & UAS & LAS & & UAS & LAS & UAS & LAS \\
\hline ar & 71.19 & 65.30 & 74.13 & 69.12 & hsb & 61.70 & 53.83 & 66.64 & 59.27 \\
\hline ar_pud & 53.55 & 43.14 & 57.18 & 48.01 & $\mathrm{hu}$ & 71.46 & 64.30 & 74.68 & 66.29 \\
\hline bg & 87.79 & 83.64 & 90.30 & 86.73 & id & 80.91 & 74.61 & 83.06 & 76.66 \\
\hline $\mathrm{bxr}^{\ddagger}$ & 46.97 & 31.50 & 46.04 & 27.66 & it & 88.03 & 85.28 & 90.05 & 87.77 \\
\hline $\mathrm{ca}$ & 88.62 & 85.39 & 90.79 & 88.27 & it_pud & 87.04 & 83.70 & 88.59 & 85.51 \\
\hline cs & 86.46 & 82.87 & 89.57 & 86.52 & ja & 73.52 & 72.21 & 81.94 & 80.85 \\
\hline cs_cac & 86.49 & 82.46 & 87.66 & 83.87 & ja_pud & 77.13 & 76.28 & 84.38 & 83.75 \\
\hline cs_cltt & 76.26 & 71.64 & 84.96 & 81.89 & $\mathrm{kk}$ & 41.92 & 24.51 & 42.11 & 24.76 \\
\hline cs_pud & 84.42 & 79.80 & 85.79 & 80.75 & $\mathrm{kmr}$ & 46.20 & 32.35 & 52.55 & 44.70 \\
\hline $\mathrm{cu}$ & 69.68 & 62.76 & 72.19 & 65.80 & ko & 66.40 & 59.09 & 76.95 & 71.82 \\
\hline $\mathrm{da}$ & 76.94 & 73.38 & 81.14 & 78.03 & la & 54.35 & 43.77 & 59.15 & 48.75 \\
\hline de & 74.27 & 69.11 & 79.03 & 74.79 & la_ittb & 80.78 & 76.98 & 84.07 & 81.03 \\
\hline de_pud & 73.64 & 66.53 & 77.90 & 71.11 & la_proiel & 63.50 & 57.54 & 68.94 & 63.48 \\
\hline el & 83.00 & 79.26 & 85.72 & 82.82 & lv & 67.14 & 59.95 & 71.91 & 64.97 \\
\hline en & 78.87 & 75.84 & 82.88 & 79.94 & $\mathrm{nl}$ & 74.94 & 68.90 & 78.90 & 73.43 \\
\hline en_lines & 77.39 & 72.94 & 82.70 & 78.73 & nl_lassysmall & 81.37 & 78.15 & 89.06 & 86.85 \\
\hline en_partut & 77.83 & 73.64 & 85.57 & 81.98 & no_bokmaal & 86.14 & 83.27 & 89.09 & 86.90 \\
\hline en_pud & 82.74 & 78.95 & 84.97 & 81.86 & no_nynorsk & 84.88 & 81.56 & 87.95 & 85.43 \\
\hline es & 84.84 & 81.47 & 87.20 & 84.22 & $\mathrm{pl}$ & 85.08 & 78.78 & 88.18 & 83.75 \\
\hline es_ancora & 86.97 & 83.78 & 89.94 & 87.39 & $\mathrm{pt}$ & 85.77 & 82.11 & 87.75 & 84.90 \\
\hline es_pud ${ }^{\ddagger}$ & 84.71 & 77.65 & 82.34 & 72.67 & pt_br & 87.75 & 85.36 & 90.51 & 88.71 \\
\hline et & 67.71 & 58.79 & 73.09 & 65.10 & pt_pud & 80.10 & 73.96 & 81.18 & 72.33 \\
\hline eu & 74.39 & 69.15 & 79.29 & 73.85 & ro & 85.50 & 79.88 & 87.30 & 82.21 \\
\hline fa & 83.36 & 79.24 & 86.24 & 82.08 & $\mathrm{ru}$ & 79.28 & 74.03 & 84.32 & 80.58 \\
\hline fi & 77.90 & 73.75 & 81.98 & 77.73 & ru_pud & 75.67 & 68.31 & 72.33 & 61.60 \\
\hline fi_ftb & 78.77 & 74.03 & 82.79 & 78.08 & ru_syntagrus & 89.30 & 86.76 & 91.71 & 89.77 \\
\hline fi_pud & 82.24 & 78.65 & 82.76 & 78.99 & sk & 78.14 & 72.75 & 84.38 & 79.82 \\
\hline fr & 84.13 & 80.75 & 86.07 & 82.67 & sl & 84.68 & 81.15 & 89.54 & 87.08 \\
\hline fr_partut & 81.69 & 77.38 & 88.39 & 84.86 & sl_sst & 53.79 & 46.45 & 60.36 & 54.06 \\
\hline fr_pud & 78.62 & 73.63 & 82.55 & 77.51 & sme & 46.06 & 30.6 & 52.51 & 38.91 \\
\hline fr_sequoia & 82.62 & 79.98 & 87.11 & 85.09 & sv & 80.78 & 76.73 & 83.93 & 80.58 \\
\hline ga & 72.08 & 61.52 & 73.48 & 61.62 & Sv_lines & 79.18 & 74.29 & 81.77 & 77.30 \\
\hline $\mathrm{gl}$ & 80.66 & 77.31 & 83.31 & 80.23 & SV_pud & 75.09 & 70.62 & 75.15 & 70.70 \\
\hline gl_treegal & 71.17 & 65.82 & 72.65 & 66.51 & $\operatorname{tr}$ & 60.48 & 53.19 & 64.14 & 56.43 \\
\hline got & 67.13 & 59.81 & 67.61 & 60.52 & tr_pud ${ }^{\ddagger}$ & 55.01 & 34.53 & 54.17 & 34.15 \\
\hline grc & 62.74 & 56.04 & 66.86 & 59.84 & $\mathrm{ug}^{\ddagger}$ & 53.58 & 34.18 & 51.57 & 34.52 \\
\hline grc_proiel & 70.42 & 65.22 & 74.19 & 69.39 & $\mathrm{uk}$ & 69.78 & 60.76 & 71.22 & 63.08 \\
\hline he & 61.54 & 57.23 & 64.30 & 60.07 & ur & 83.67 & 76.69 & 86.41 & 79.72 \\
\hline hi & 90.97 & 86.77 & 93.31 & 89.48 & vi & 42.12 & 37.47 & 47.53 & 42.52 \\
\hline hi_pud & 63.43 & 50.85 & 67.24 & 54.14 & $\mathrm{zh}$ & 61.5 & 57.40 & 68.95 & 65.10 \\
\hline $\mathrm{hr}$ & 83.20 & 77.18 & 86.58 & 81.30 & Average & 74.41 & 68.35 & 77.81 & 72.11 \\
\hline
\end{tabular}

Table 7: End-to-end parsing results on all test treebanks. ${ }^{*}$ indicates the test sets on which UDPipe performs better. Among the 5 sets, es_pud, ru_pud and tr_pud are parallel test sets on which we simply use the model trained from the source treebank. We suggest better strategies should be explored.

\begin{tabular}{l|cl|ll|ll}
\hline \multirow{2}{*}{ Itcode } & \multicolumn{2}{|c|}{ Ours (b/r) } & \multicolumn{2}{c|}{ Ours (a/r) } & \multicolumn{2}{c}{ Rank-1 } \\
& WSeg & LAS & WSeg & LAS & WSeg & LAS \\
\hline ja & 92.95 & 80.85 & 94.70 & 84.37 & $\mathbf{9 8 . 5 9}$ & $\mathbf{9 1 . 1 3}$ \\
ja_pud & 94.02 & 83.75 & $\mathbf{9 5 . 5 4}$ & $\mathbf{8 5 . 3 3}$ & 94.93 & 83.75 \\
vi & 84.70 & 42.52 & $\mathbf{9 1 . 4 0}$ & $\mathbf{4 8 . 9 8}$ & 87.30 & 47.51 \\
zh & 91.19 & 65.10 & $\mathbf{9 5 . 2 1}$ & $\mathbf{7 0 . 4 9}$ & 94.57 & 68.56 \\
\hline
\end{tabular}

Table 8: Post-evaluation results on zh, vi, ja and ja_pud. b/r: before revision. a/r: after revision.

Jan Mašek, Yuji Matsumoto, Ryan McDonald, Gustavo Mendonça, Anna Missilä, Verginica Mititelu, Yusuke Miyao, Simonetta Montemagni, Amir More, Laura Moreno Romero, Shunsuke Mori, Bohdan Moskalevskyi, Kadri Muischnek, Nina Mustafina, Kaili Müürisep, Pinkey Nain- wani, Anna Nedoluzhko, Lng Nguyn Th, Huyn Nguyn Th Minh, Vitaly Nikolaev, Rattima Nitisaroj, Hanna Nurmi, Stina Ojala, Petya Osenova, Lilja Øvrelid, Elena Pascual, Marco Passarotti, CenelAugusto Perez, Guy Perrier, Slav Petrov, Jussi Piitulainen, Emily Pitler, Barbara Plank, Martin Popel, Lauma Pretkalnia, Prokopis Prokopidis, Tiina Puolakainen, Sampo Pyysalo, Alexandre Rademaker, Livy Real, Siva Reddy, Georg Rehm, Larissa Rinaldi, Laura Rituma, Rudolf Rosa, Davide Rovati, Shadi Saleh, Manuela Sanguinetti, Baiba Saulite, Yanin Sawanakunanon, Sebastian Schuster, Djamé Seddah, Wolfgang Seeker, Mojgan Seraji, Lena Shakurova, Mo Shen, Atsuko Shimada, Muh Shohibussirri, Natalia Silveira, Maria Simi, Radu Simionescu, Katalin Simkó, Mária Šimková, Kiril Simov, Aaron Smith, Antonio Stella, Jana Str- 
nadová, Alane Suhr, Umut Sulubacak, Zsolt Szántó, Dima Taji, Takaaki Tanaka, Trond Trosterud, Anna Trukhina, Reut Tsarfaty, Francis Tyers, Sumire Uematsu, Zdeňka Urešová, Larraitz Uria, Hans Uszkoreit, Gertjan van Noord, Viktor Varga, Veronika Vincze, Jonathan North Washington, Zhuoran Yu, Zdeněk Žabokrtský, Daniel Zeman, and Hanzhi Zhu. 2017a. Universal dependencies 2.0 CoNLL 2017 shared task development and test data. LINDAT/CLARIN digital library at the Institute of Formal and Applied Linguistics, Charles University. http://hdl.handle.net/11234/1-2184.

Joakim Nivre, Željko Agić, Lars Ahrenberg, Maria Jesus Aranzabe, Masayuki Asahara, Aitziber Atutxa, Miguel Ballesteros, John Bauer, Kepa Bengoetxea, Riyaz Ahmad Bhat, Eckhard Bick, Cristina Bosco, Gosse Bouma, Sam Bowman, Marie Candito, Gülşen Cebirolu Eryiit, Giuseppe G. A. Celano, Fabricio Chalub, Jinho Choi, Çar Çöltekin, Miriam Connor, Elizabeth Davidson, Marie-Catherine de Marneffe, Valeria de Paiva, Arantza Diaz de Ilarraza, Kaja Dobrovoljc, Timothy Dozat, Kira Droganova, Puneet Dwivedi, Marhaba Eli, Tomaž Erjavec, Richárd Farkas, Jennifer Foster, Cláudia Freitas, Katarína Gajdošová, Daniel Galbraith, Marcos Garcia, Filip Ginter, Iakes Goenaga, Koldo Gojenola, Memduh Gökrmak, Yoav Goldberg, Xavier Gómez Guinovart, Berta Gonzáles Saavedra, Matias Grioni, Normunds Grūzitis, Bruno Guillaume, Nizar Habash, Jan Hajič, Linh Hà M, Dag Haug, Barbora Hladká, Petter Hohle, Radu Ion, Elena Irimia, Anders Johannsen, Fredrik Jørgensen, Hüner Kaşkara, Hiroshi Kanayama, Jenna Kanerva, Natalia Kotsyba, Simon Krek, Veronika Laippala, Phng Lê Hng, Alessandro Lenci, Nikola Ljubešić, Olga Lyashevskaya, Teresa Lynn, Aibek Makazhanov, Christopher Manning, Cătălina Mărănduc, David Mareček, Héctor Martínez Alonso, André Martins, Jan Mašek, Yuji Matsumoto, Ryan McDonald, Anna Missilä, Verginica Mititelu, Yusuke Miyao, Simonetta Montemagni, Amir More, Shunsuke Mori, Bohdan Moskalevskyi, Kadri Muischnek, Nina Mustafina, Kaili Müürisep, Lng Nguyn Th, Huyn Nguyn Th Minh, Vitaly Nikolaev, Hanna Nurmi, Stina Ojala, Petya Osenova, Lilja Øvrelid, Elena Pascual, Marco Passarotti, Cenel-Augusto Perez, Guy Perrier, Slav Petrov, Jussi Piitulainen, Barbara Plank, Martin Popel, Lauma Pretkalnia, Prokopis Prokopidis, Tiina Puolakainen, Sampo Pyysalo, Alexandre Rademaker, Loganathan Ramasamy, Livy Real, Laura Rituma, Rudolf Rosa, Shadi Saleh, Manuela Sanguinetti, Baiba Saulite, Sebastian Schuster, Djamé Seddah, Wolfgang Seeker, Mojgan Seraji, Lena Shakurova, Mo Shen, Dmitry Sichinava, Natalia Silveira, Maria Simi, Radu Simionescu, Katalin Simkó, Mária Šimková, Kiril Simov, Aaron Smith, Alane Suhr, Umut Sulubacak, Zsolt Szántó, Dima Taji, Takaaki Tanaka, Reut Tsarfaty, Francis Tyers, Sumire Uematsu, Larraitz Uria, Gertjan van Noord, Viktor Varga, Veronika Vincze, Jonathan North Washington, Zdeněk Žabokrtský,
Amir Zeldes, Daniel Zeman, and Hanzhi Zhu. 2017b. Universal Dependencies 2.0. LINDAT/CLARIN digital library at the Institute of Formal and Applied Linguistics, Charles University, Prague, http://hdl.handle.net/ 11234/1-1983. http://hdl.handle.net/11234/11983.

Joakim Nivre, Marie-Catherine de Marneffe, Filip Ginter, Yoav Goldberg, Jan Hajič, Christopher Manning, Ryan McDonald, Slav Petrov, Sampo Pyysalo, Natalia Silveira, Reut Tsarfaty, and Daniel Zeman. 2016. Universal Dependencies v1: A multilingual treebank collection. In Proceedings of the 10th International Conference on Language Resources and Evaluation (LREC 2016). European Language Resources Association, Portoro, Slovenia, pages 16591666.

Martin Potthast, Tim Gollub, Francisco Rangel, Paolo Rosso, Efstathios Stamatatos, and Benno Stein. 2014. Improving the reproducibility of PAN's shared tasks: Plagiarism detection, author identification, and author profiling. In Evangelos Kanoulas, Mihai Lupu, Paul Clough, Mark Sanderson, Mark Hall, Allan Hanbury, and Elaine Toms, editors, Information Access Evaluation meets Multilinguality, Multimodality, and Visualization. 5th International Conference of the CLEF Initiative (CLEF 14). Springer, Berlin Heidelberg New York, pages 268-299. https://doi.org/10.1007/978-3-31911382-1_22.

Milan Straka, Jan Hajic, and Jana Straková. 2016a. Ud-pipe: Trainable pipeline for processing conll-u files performing tokenization, morphological analysis, pos tagging and parsing. In Proceedings of the Tenth International Conference on Language Resources and Evaluation (LREC 2016).

Milan Straka, Jan Hajič, and Jana Straková. 2016b. UDPipe: trainable pipeline for processing CoNLL$\mathrm{U}$ files performing tokenization, morphological analysis, POS tagging and parsing. In Proceedings of the 10th International Conference on Language Resources and Evaluation (LREC 2016). European Language Resources Association, Portoro, Slovenia.

Oscar Täckström, Ryan McDonald, and Jakob Uszkoreit. 2012. Cross-lingual word clusters for direct transfer of linguistic structure. In NAACL. Association for Computational Linguistics, Montréal, Canada, pages 477-487.

Kai Sheng Tai, Richard Socher, and Christopher D. Manning. 2015. Improved semantic representations from tree-structured long short-term memory networks. In Proc. of ACL and IJCNLP. pages 15561566.

Peilu Wang, Yao Qian, Frank K. Soong, Lei He, and Hai Zhao. 2016. Learning distributed word representations for bidirectional lstm recurrent neural network. In Proc. of HLT-NAACL. 
David Weiss, Chris Alberti, Michael Collins, and Slav Petrov. 2015. Structured training for neural network transition-based parsing. CoRR abs/1506.06158. http://arxiv.org/abs/1506.06158.

Daniel Zeman, Martin Popel, Milan Straka, Jan Hajič, Joakim Nivre, Filip Ginter, Juhani Luotolahti, Sampo Pyysalo, Slav Petrov, Martin Potthast, Francis Tyers, Elena Badmaeva, Memduh Gökırmak, Anna Nedoluzhko, Silvie Cinková, Jan Hajič jr., Jaroslava Hlaváčová, Václava Kettnerová, Zdeňka Urešová, Jenna Kanerva, Stina Ojala, Anna Missilä, Christopher Manning, Sebastian Schuster, Siva Reddy, Dima Taji, Nizar Habash, Herman Leung, Marie-Catherine de Marneffe, Manuela Sanguinetti, Maria Simi, Hiroshi Kanayama, Valeria de Paiva, Kira Droganova, Hěctor Martínez Alonso, Hans Uszkoreit, Vivien Macketanz, Aljoscha Burchardt, Kim Harris, Katrin Marheinecke, Georg Rehm, Tolga Kayadelen, Mohammed Attia, Ali Elkahky, Zhuoran Yu, Emily Pitler, Saran Lertpradit, Michael Mandl, Jesse Kirchner, Hector Fernandez Alcalde, Jana Strnadova, Esha Banerjee, Ruli Manurung, Antonio Stella, Atsuko Shimada, Sookyoung Kwak, Gustavo Mendonça, Tatiana Lando, Rattima Nitisaroj, and Josie Li. 2017. CoNLL 2017 Shared Task: Multilingual Parsing from Raw Text to Universal Dependencies. In Proceedings of the CoNLL 2017 Shared Task: Multilingual Parsing from Raw Text to Universal Dependencies. Association for Computational Linguistics.

Xiaodan Zhu, Parinaz Sobihani, and Hongyu Guo. 2015. Long short-term memory over recursive structures. In Proc. of ICML. pages 1604-1612. 